\title{
Epirubicin: a new entry in the list of fetal cardiotoxic drugs? Intrauterine death of one fetus in a twin pregnancy. Case report and review of literature
}

Marialuisa Framarino-dei-Malatesta ${ }^{1 *}$, Giuseppina Perrone ${ }^{1}$, Antonella Giancotti ${ }^{1}$, Flavia Ventriglia ${ }^{2}$, Martina Derme $^{1}$, Isabella lannini ${ }^{1}$, Valentina Tibaldi ${ }^{1}$, Paola Galoppi ${ }^{1}$, Paolo Sammartino ${ }^{3}$, Gianluca Cascialli ${ }^{1}$ and Roberto Brunelli ${ }^{1}$

\begin{abstract}
Background: Current knowledge indicate that epirubicin administration in late pregnancy is almost devoid of any fetal cardiotoxicity. We report a twin pregnancy complicated by breast cancer in which epirubicin administration was causatively linked to the death of one twin who was small for gestational age (SGA) and in a condition of oligohydramnios and determined the onset of a transient cardiotoxicity of the surviving fetus/newborn.

Case presentation: A 38-year-old caucasic woman with a dichorionic twin pregnancy was referred to our center at 20 and 1/7 weeks for a suspected breast cancer, later confirmed by the histopathology report. At 31 and 3/7 weeks, after the second chemotherapy cycle, ultrasound examination evidenced the demise of one twin while cardiac examination revealed a monophasic diastolic ventricular filling, i.e. a diastolic dysfunction of the surviving fetus who was delivered the following day due to the occurrence of grade II placental abruption. The role of epirubicin cardiotoxicity in the death of the first twin was supported by post-mortem cardiac and placental examination and by the absence of structural or genomic abnormalities that may indicate an alternative etiology of fetal demise. The occurrence of epirubicin cardiotoxicity in the surviving newborn was confirmed by the report of high levels of troponin and transient left ventricular septal hypokinesia.

Conclusion: Based on our findings we suggest that epirubicin administration in pregnancy should be preceded by the screening of some fetal conditions like SGA and oligohydramnios that may increase its cardiotoxicity and that, during treatment, the diastolic function of the fetal right ventricle should be specifically monitored by a pediatric cardiologist; also, epirubicin and desamethasone for lung maturation should not be closely administered since placental effects of glucocorticoids may increase epirubicin toxicity.
\end{abstract}

Keywords: Epirubicin, Cardiotoxicity, Twin pregnancy, Fetal death, Breast cancer in pregnancy

\section{Background}

The rapidly changing sociocultural and epidemiological scene seems to increase in the near future the incidence of breast cancer in pregnancy (BCP). Recent years have witnessed a rising age at childbearing in Western countries. In Italy the mean age at first delivery increased from 29.8 years in 1995 to 31.5 years in 2013

\footnotetext{
* Correspondence: marialuisa.framarino@gmail.com

${ }^{1}$ Department of Gynecologic Obstetrics and Urology Sciences, University of Rome "Sapienza", Rome, Italy

Full list of author information is available at the end of the article
}

[1]. At the same time, age at breast cancer onset in Italy has reportedly decreased, and the incidence rates for breast cancer in non-pregnant women under 45 years increased from 20.06 per 100,000 in 1980 to 32.85 per 100,000 in 2015 [2]. The increasing age at childbearing and younger age at breast cancer onset therefore imply an increased risk of BCP.

Therapeutic approaches in BCP depend on tumor stage, tumor biology, gestational age and patient's wishes. Systemic chemotherapy may be required before or after surgery, and benefits for the mother must be 
compared with the potential harm to the fetus from in utero exposure to chemotherapeutics. The adhesion of patients with BCP to standard protocols based on the administration of anthracyclines/alkylating agents is highly recommended [3] as it grants patients with BCP the same disease-free interval and overall survival rates observed for non-pregnant patients with the same stage of disease $[4,5]$.

All chemotherapeutics are potentially teratogenic or may induce toxicity and organ dysfunction in the fetus but current knowledge indicate that anthracyclines including epirubicin administration in late pregnancy is almost devoid of any fetal cardiotoxicity.

We report a case of dichorionic pregnancy complicated by breast cancer, in which epirubicin administration was associated to the death of one twin and to the contemporary evidence of a reversible cardiotoxicity of the surviving fetus/newborn.

\section{Case presentation}

A 38-year-old caucasic woman, G1P0, with a dichorionic twin pregnancy was referred to our center at 20 and $1 / 7$ weeks for an excisional breast biopsy due to a suspected breast cancer. The patient underwent right external quadrantectomy with first level lymphnode (LN) dissection. Neither family history for breast cancer nor previous surgical interventions were reported. The pathology report showed an invasive and poorly differentiated (G3) ductal carcinoma not otherwise specified (NOS) measuring $2 \mathrm{~cm}$ in diameter (pT1). Examined LN (10) were negative for metastasis. The immunohistochemical evaluation [absent estrogen receptors (ER $0 \%$ ) and C-erb-Neu expression, positive progesteron receptors (PgR 40 \%), and Ki-67 67 \%] suggested a high risk of relapse, prompting the start of adjuvant chemotherapy. Maternal echocardiogram and laboratory tests were all within the normal range. A chemotherapy regimen based on epirubicin $90 \mathrm{mg} / \mathrm{m} 2$ and cyclophosphamide $600 \mathrm{mg} / \mathrm{m} 2$ was started at a gestational age of 27 and 0/7 weeks; overall, the patient received 2 cycles of chemotherapy on a 21 days outpatient basis.

A complete assessment of fetal well-being, including the combined evaluations of fetal heart rate short term variation (STV), the largest vertical pocket of amniotic fluid (LVP-AF), pulsatility indices of the umbilical artery (UA-PI), middle cerebral artery (MCA-PI) and ductus venosus (DV-PIV), was performed before the start of chemotherapy and weekly thereafter; control of fetal growth pattern was scheduled every 3 weeks.

At baseline fetal ultrasound evaluation (26 and 6/7 weeks), one twin (A) displayed normal anatomy and was scored as small for gestational age (SGA), due to an estimated fetal weight $(\mathrm{EFW})<10$ percentile for gestational age $(679 \mathrm{gr})$ in the absence of signs of chronic placental dysfunction including fetal circulatory redistribution (UA-PI: 1 and MCA-PI: 1.5) and/or abnormal Doppler analysis of the uterine arteries (mean resistence index: 0.4); for twin A a condition of oligohydramnios was also evidenced (LVPAF: $15 \mathrm{~mm}$ ).

Fetal surveillance was completely unremarkable for both twin A and B during the two weeks that followed the first cycle of chemotherapy. At 31 and $0 / 7$ weeks, immediately after the second chemotherapy cycle, all parameters of twin B were scored as normal. Twin A presented an unaltered growth pattern (EFW $<10$ percentile) and a persistent oligohydramnios (LVP-AF of $12 \mathrm{~mm}$ ) along with normal UA-PI (1.19), MCA-PI (1.6) and STV (6.3 msec); of note, right cardiac sections appeared dilated and an abnormally high DV-PIV (1.1) was recorded, although with no evidence of reverse flow (Fig. 1 Panel a). Antenatal corticosteroids were administered (desamethasone $12 \mathrm{mg}, 24 \mathrm{~h}$ apart). The following day (31 and 1/ 7 weeks), STV values were normal (5.9 and $6.3 \mathrm{msec}$ for twins $A$ and $B$, respectively).

At 31 and $3 / 7$ weeks, ultrasounds evidenced the demise of twin A; an echocardiogram of twin B showed a monophasic diastolic filling addressing a diastolic dysfunction of the right ventricle, together with a mild hypokinesia of the ventricular septum (Fig. 1 Panel b).

At 31 and 4/7 weeks of gestation, twin B (2028 gr) was delivered by emergency cesarean section due to grade II placental abruption (Apgar score $7 / 9$ at 1 and $5 \mathrm{~min}$, respectively).

The autopsy of twin A (900 g) did not evidence structural abnormalities; cardiac histology showed severe myocardial interstitial edema with fiber dissociation and sporadic vacuolar myocyte degeneration (Fig. 1 Panel c). The only other remarkable findings were found in twin A extravillous throphoblast (hypertrophic vacuolization and nuclear pleomorphism) and chorionic villi (interstitial edema and fibrinoid necrosis) (Fig. 1 Panel D). Comparative genomic hybridization $(\mathrm{CGH})$ analysis, performed on umbilical cord extracted DNA, did not show any genomic rearrangement.

Routine laboratory tests obtained for twin B during the first day of life (DOL) were normal; however, a documented significant septal hypokinesia (Fig. 1 Panel e), confirmed by a pediatric cardiologist with 20 years of experience in perinatal cardiac assessment, was associated to extremely high concentrations of troponin (Tn1) (Tn1: $0.21 \mu \mathrm{g} / \mathrm{L}$; reference $<0.0014 \mu \mathrm{g} / \mathrm{L})$; Tn1 was still abnormally elevated on DOL 19 (Tn1: $0.06 \mu \mathrm{g} / \mathrm{L}$ ) but progressively decreased back to normal values on DOL 40. Pediatric cardiology examinations were regularly performed (every four weeks) up to six months of age and confirmed regular post-natal cardiac function.

Overall, the causative role of epirubicin cardiotoxicity in the death of twin $\mathrm{A}$ is supported by various evidences 

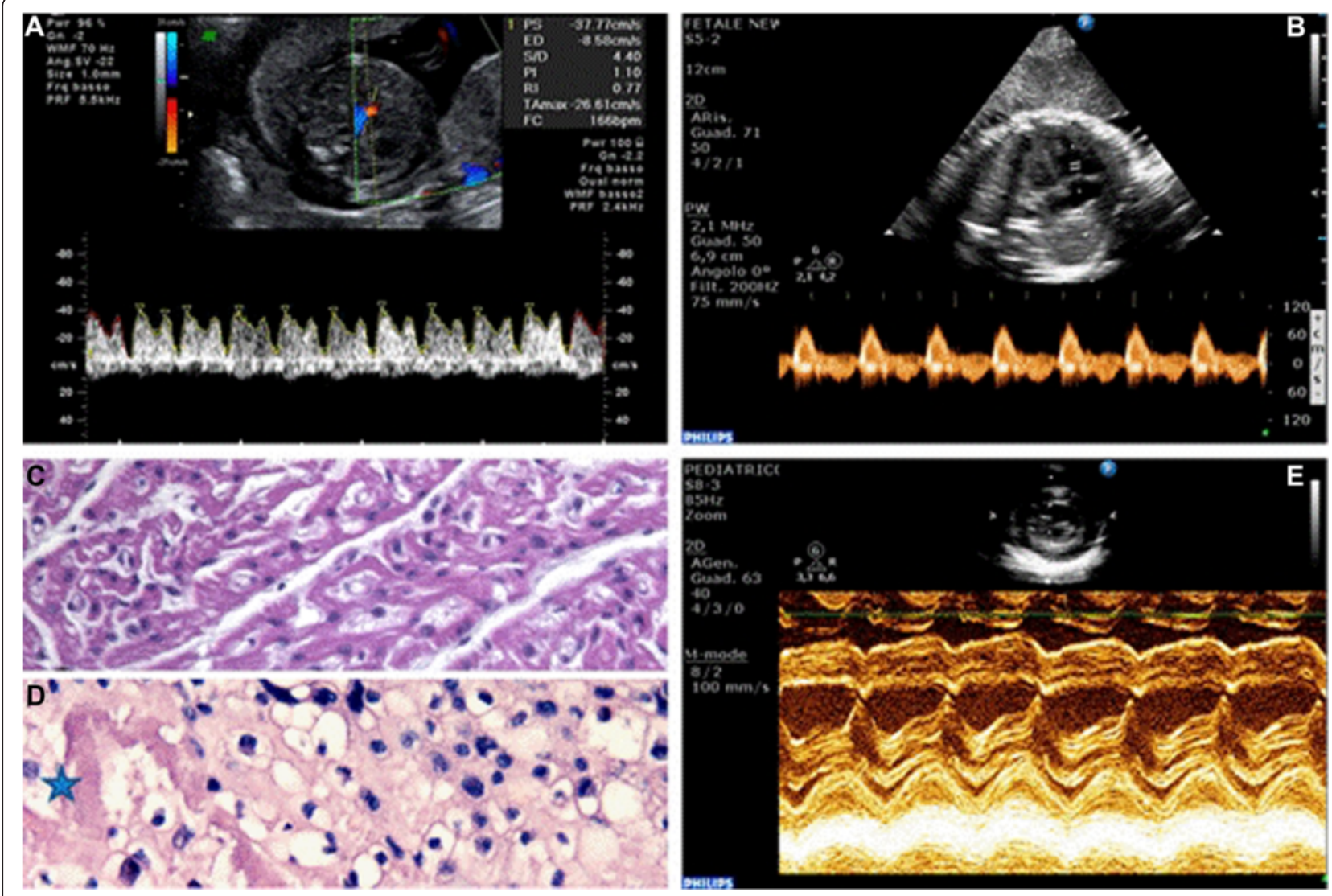

Fig. 1 Panel a: Sonogram showing a significantly elevated DV-PIV in twin A. Panel b: Echocardiogram of twin B. Four chamber view. PW Doppler of flow through tricuspidal valve. Monofasic diastolic filling of the right ventricle; hallmark of diastolic function. Panel c: Myocardial severe interstitial edema with fiber dissociation and sporadic vacuolar myocyte degeneration of the twin A fetal heart. Panel d: Hypertrophic vacuolization and nuclear pleomorphism of extravillous throphoblast, with interstitial edema and areas of fibrinoid necrosis of placenta (*). Panel e: Neonatal echocardiography of twin B. M-mode long axis of the left ventricle. Evidence of mild septal hypokinesia with an overall preserved global contractility

including: 1 - the post-mortem cardiac examination revealing the hallmarks of subacute anthracycline toxicity, i.e. massive interstitial edema without cellular infiltrates and myofibrillar damage/vacuolization [6]; 2- the histologic findings in both chorionic villi and extravillous throphoblast, well fitting the described placental effects of anthracycline exposure [7]; 3- the manifestation of acute myocardial diastolic dysfunction, evidenced by the enlarged right cardiac chambers and an elevated ductus venosus pulsatility index, preceding fetal death; 4 - the absence of structural or genomic abnormalities that may indicate an alternative etiology of fetal demise. Of note, neither arterial Doppler nor STV evaluation anticipated the impending fetal demise.

\section{Conclusions}

The first trimester is the most critical time regarding teratogenic effects. The blastocyst is resistant to teratogenic drugs in the first 2 weeks from conceptions whereas the administration of chemotherapy during organogenesis from 4 to 13 weeks of pregnancy is associated with an increased risk of miscarriage or congenital malformations [8] as largely documented from case reports, case series and collected reviews [9-11]. Second and third trimester chemotherapeutics exposure after the end of organogenesis, does not usually increase the teratogenic risk but may cause neurocognitive development disorders and increasing risk of intrauterine growth retardation (IUGR), pre-term labour and low birth weight [12-16].

According to SOGC guidelines, we should administer the standard regimens based on a combination of anthracyclines/alkylating agents after the end of the first trimester [17].

The first prospective collection of data addressing the issue of antracyclines safety profile in pregnancy was first reported by the Texas MD Anderson Cancer Center back in 1999. These authors treated 24 pregnant patients with primary or recurrent cancer of the breast managed with a standardized protocol of 5-fluorouracil + doxorubicin + cyclophosphamide (FAC) chemotherapy in the second and third trimester of pregnancy and did not report an increased rate of congenital anomalies [18]. In 
the same year, a French survey reporting 12 patients with BCP alternatively treated with FAC, 5-fluorouracil + epirubicin + cyclophosphamide (FEC), epirubicin + cyclophosphamide (EC) or doxorubicin + cyclophosphamide (AC) evidenced only one case of intrauterine death at 30 weeks of gestation [19]. In a retrospective, cohort study evaluating the fetal risks involved in the administration of cancer chemotherapy during gestation, one fetus died after second trimester exposure to epirubicin, vincristine and prednisone but no malformation was detected [20]. Hahn et al. extended the previous evidences from the MD Anderson Cancer Center and confirmed the absence of congenital birth defects in fetuses exposed to anthracyclines chemotherapy in utero; indeed, only three children reported congenital malformations in a group of 57 women treated up to 2006, with a median number of four FAC cycles given during pregnancy; one neonate was born with Down syndrome, one with ureteral reflux, and a third with club foot [21]. Ring et al. evaluated 16 out of 28 BCP patients receiving anthracyclines-based chemotherapy during pregnancy without reporting any congenital birth defect [22].

The German Breast Group issued the first International Recommendations on $\mathrm{BCP}$ and confirmed the safety of anthracyclines [23]. An international consensus meeting held in 2010 confirmed that anthracyclines can be used in the setting of BCP [24] and RCOG guidelines assigned an Evidence level 3 to the statement that anthracycline-based chemotherapy in the second and third trimesters can be administered with minimal risk to the developing fetus [25].

Anthacyclines display well-known cardiotoxic effects: age, cumulative dose and previous radiotherapy increases the rates of cardiac damage in children and adults [26]. The molecular mechanisms underlying antracyclines cardiotoxicity are not fully understood, but include alterations of cell membranes fluidity and ion transport with generation of reactive oxygen species by ironanthracycline complexes, leading to lipid peroxidation and membrane damage [27] and the impairment of DNA repair through the interaction with the topoisomerase-IIbeta enzyme in myocytes [28]. Increasing evidences show that the extracellular matrix plays a complex and diverse role in some processes initiated by anthracyclines that finally lead to cardiac damage [29]. Notably, fetal myocardium is theoretically more vulnerable to damage by chemotherapeutics because fetal myocytes are smaller than adult ones, and contain fewer sarcomeres and mitochondria [30].

Fetal safety during the administration of anthracyclinebased chemotherapy in pregnancy is of theoretical concern because anthracyclines can cross the placenta, even if their fetal plasma concentrations are lower than those found in the mother, and have cumulative toxicity
[31]. Available data provide only limited experimental and clinical data on the transplacental transfer of these chemotherapeutics in pregnant women; in a baboon model, fetal plasma concentrations of doxorubicin, epirubicin and paclitaxel were about $7.5 \%, 4.0 \%$, and $1.4 \%$, of the respective maternal concentrations [32]. Fetal blood samples from pregnant rats receiving doxorubicin showed a plasmatic concentration that was $6.2 \%$ that of the mother; interestingly, neither Doppler analysis nor heart microstructure or cellular DNA turnover and apoptosis were influenced by doxorubicin exposure [33].

Owing to the molecular weight of doxorubicin is 580 dalton, there is an incomplete transfer of the drug across the placental barrier [8]. However, the transplacental passage cannot be simply predicted from the physicalchemical properties of the drugs like the molecular weight. Really, while assessing fetal plasma drug concentrations, the functional expression of many members of the ATP-binding cassette $(\mathrm{ABC})$ efflux transporters that are highly expressed in the human placenta, should be adequately considered; indeed, these transporters prevent the trans-placental transfer of cytotoxic compounds present in the maternal circulation, therefore protecting the fetus [34, 35]; specifically, anthracyclines and taxanes are substrates for $\mathrm{ABC}$-transporters like the major placental drug-transporting P-glycoprotein, that keeps low the fetal plasma concentrations of these harmful compounds [36].

Anthracyclines do not collectively share the same low rate of transplacental transfer. Indeed, idarubicin, being more lipophilic than other antracyclines, easily crosses the placenta; Germann reported one fetal death and one case of reversible heart dysfunction in a group of patients affected by acute myeloid leukemia receiving idarubicin-based chemotherapy during the third trimester of pregnancy [37]. Similarly, Baumgartner reported one case of reversible fetal cardiomyopathy following the use of idarubicin during pregnancy [38] while the occurrence of a severe idarubicin-related cardiotoxicity in a newborn was described in a swiss study [39]. Altogether, these findings suggest a close fetal monitoring during idarubicin based chemotherapy; long-term outcomes of idarubicin exposed children need further investigations.

Unlike idarubicin, doxorubicin and epirubicin, due to their low levels in fetal plasma, may be administered during the second and third trimesters without significant risk of fetal myocardial dysfunction. At first, clinicians gained some experience on the safety profile of these two antracyclines from case reports and small case series [40]. Further reassuring evidence was granted by an Italian review reporting that only 13/out of 403 (3\%) children exposed to these anthracyclines during late pregnancy developed short-term cardiac complications [41]. Azim et al. reported that different epirubicin and 
doxorubicin regimens administered in adjuvant, neoadjuvant and metastatic settings (23 patients and 3 patients, respectively) did not adversely affect the course of pregnancy or fetal/neonatal outcome [42]. In a small cohort of patients, even a dose-dense antracyclines chemotherapy administered every two weeks did not involve a higher risk of fetal complications [43].

In a prospective case-control clinical study, Gziri found that maternal and fetal cardiac functions were not significantly hampered by anthracyclines exposure in pregnancy but rather displayed only minor changes of the myocardial performance index and the tricuspid inflow devoid of any clinical relevance [44].

Notably, epirubicin in pregnancy has a shorter terminal half-life than doxorubicin due to its combined glucuronization by the liver and the placenta [45] and therefore displays a better therapeutic index with fewer systemic and cardiotoxic effects. In an Austrian study, all three patients managed at the University Hospital of Vienna with six courses of FEC neoadjuvant chemotherapy delivered healthy newborns [46]. Others case reports on multidrugs regimens including epirubicin as adjuvant treatment for pregnant women with high-risk breast cancer failed to show any fetal cardiotoxicity [47, 48].

Some authors report that weekly epirubicin schedule seems particularly safe because it decreases the potential adverse events and simultaneously facilitates a close monitoring of pregnancy [49].

Overall, anthracyclines emerge as theoretically safe during the late trimesters of pregnancy, fetal concentrations being 100/1000-fold lower than adults as a result of the high molecular weight, the hydrophilic charge leading to a limited transplacental passage and the active clearance operated by the placental P-glycoprotein transporter. Indeed, despite the difficulty of comparing different agents and schedules used for BCP, fetal cardiotoxicity never emerged as a major problem of anthracyclines administration; in particular the available evidences indicate that epirubicin harmful effects on fetal heart are very limited with only one reported case of transient ventricular hypokinesia [41].

In this otherwise quite reassuring scenario, we provide evidence that, in a twin pregnancy complicated by breast cancer, epirubicin administration was causatively linked to the death of one twin and to the onset of a reversible cardiotoxicity of the surviving fetus/newborn.

The ultimate cause of twin A great susceptibility to the cardiotoxic action of epirubicin remains elusive. Anthracyclines are concentrated up to nine times more in the amniotic fluid than in fetal plasma [32]; in this regard, the presence of oligohydramnios and the histologic evidences of altered extravillous throphoblast and chorionic villi, suggest the a putative contribution of an abnormal amniotic fluid dynamics to the increased/ prolonged toxicity of epirubicin in twin A. Further support to the hypothesis of an hampered epirubicin farmacokynetics is offered by the circumstantial evidences that the succumbing SGA twin A died shortly after the administration for lung maturation of glucocorticoids; these steroids, among a myriad of actions, are known to downregulate the throphoblast expression of the detoxifying P-glycoprotein transporter [50]. Epirubicin cardiotoxicity was also evident, although to a lesser extent, in Twin B as shown by 1 - the prenatal findings of an isolated mild right ventricular diastolic dysfunction (reflecting the greater after load of this ventricle in the fetal circulation) and 2- postnatal recording of increased Tn1, associated to a transient left ventricular septal hypokinesia [51].

In conclusion, with reference to the above mentioned considerations, we suggest that a precautional use of epirubicin in pregnancy should include; 1 - the screening of oligohydramnios since this condition may putatively increase epirubicin cardiotoxicity 2- a timely surveillance by a pediatric cardiologist of the diastolic function of the fetal right ventricle, because the other indices of fetal well being are poorly predictive of an impending fetal cardiac decompensation 3- the avoidance of a close administration of epirubicin and desamethasone since glucocorticoids may hamper placental metabolism of epirubicin, ultimately increasing its toxicity.

\section{Consent to publish}

Written informed consent was obtained from the patient for publication of this Case report and any accompanying images. A copy of the written consent is available for review by the Editor of this journal.

\section{Competing interests}

The authors declare that they have no competing interests.

\section{Authors' contributions}

MFdM conceived the study, drafted and revised the manuscript, performed the analysis of data and was one of the attending physician of the patient. GP substantially contributed to the design and revision of the manuscript. AG substantially contributed to the acquisition of data and critically revised the manuscript. FV substantially contributed to the acquisition of data and critically revised the manuscript. MD analyzed and interpretated data and critically revised the manuscript. II analyzed and interpretated data and critically revised the manuscript. VT analyzed and interpretated data and drafted the manuscript. PG substantially contributed to the design and revision of the manuscript. PS analyzed and interpretated data and drafted the manuscript. GC analyzed and interpretated data and critically revised the manuscript. RB conceived the study, drafted and revised the manuscript, performed the analysis of data and was one of the attending physician of the patient. All Authors read and approved the final manuscript. All Authors read and approved the final manuscript.

Acknowledgements

There are no acknowledgements to be mentioned. 


\section{Author details}

'Department of Gynecologic Obstetrics and Urology Sciences, University of Rome "Sapienza", Rome, Italy. ${ }^{2}$ Department of Pediatrics, University of Rome "Sapienza", Rome, Italy. ${ }^{3}$ Department of Surgery "Pietro Valdoni", University of Rome "Sapienza", Rome, Italy.

Received: 23 July 2015 Accepted: 5 December 2015

Published online: 16 December 2015

\section{References}

1. Fecondità in ripresa e calendario riproduttivo posticipato. National Institute of Statistics 2013. http://noi-italia.istat.it. Accessed 30 June 2015.

2. I tumori in Italia. National Institute of Health. http://www.tumori.net. Accessed 18 May 2015.

3. Kaklamani VG, Gradishar WJ (2003) Epirubicin versus doxorubicin: which is the anthracycline of choice for the treatment of breast cancer? Clin Breast Cancer 4(Suppl 1):S26-S33

4. Framarino Dei Malatesta M, Piccioni MG, Brunelli R, lannini I, Cascialli G, Sammartino P (2014) Breast cancer during pregnancy: a retrospective study on obstetrical problems and survival. Eur J Obstet Gynecol Reprod Biol 173:48-52

5. Litton JK, Warneke CL, Hahn KM, Palla SL, Kuerer HM, Perkins GH et al (2013) Case control study of women treated with chemotherapy for breast cancer during pregnancy as compared with nonpregnant patients with breast cancer. Oncologist 18:369-76

6. Hengel CL, Russel PA, Gould PA, Kaye DM (2006) Subacute anthracycline cardiotoxicity. Heart Lung Circ 15:59-61

7. Abellar RG, Pepperell JR, Greco D, Gundogan F, Kostadinov S, Schwartz J et al (2009) Effects of chemotherapy during pregnancy on the placenta. Pediatric Dev Pathol 12:35-41

8. Koren G (2011) Fetal risks of maternal pharmacotherapy: identifying signals. Handb Exp Pharmacol 205:285-94

9. Cardonick $\mathrm{E}$, lacobucci $\mathrm{A}$ (2004) Use of chemotherapy during human pregnancy. Lancet Oncol 5:283-91

10. Nurmohamed L, Moretti ME, Schechter T, Einarson A, Johnson D, Lavigne SV et al (2011) Outcome following high-dose methotrexate in pregnancies misdiagnosed as ectopic. Am J Obstet Gynecol 205:533-e1-3

11. Leyder M, Laubach M, Breugelmans M, Keymolen K, De Greve J, Foulon W (2011) Specific congenital malformations after exposure to cyclophosphamide, epirubicin and 5-fluorouracil during the first trimester of pregnancy. Gynecol Obstet Invest 71:141-4

12. Berretta M, Di Francia R, Lleshi A, De Paoli P, Li Volti G, Bearz A et al (2012) Antiblastic treatment, for solid tumors, during pregnancy: a crucial decision. Int J Immunopathol Pharmacol 25(2 Suppl):1S-19S

13. Abdel-Hady E-S, Hemida RA, Gamal A, El-Zafarany M, Toson E, El-Bayoumi MA (2012) Cancer during pregnancy: perinatal outcome after in utero exposure to chemotherapy. Arch Gynecol Obstet 286:283-6

14. Amant F, Loibl S, Neven P, Van Calsteren K (2012) Breast cancer in pregnancy. Lancet 379:570-9

15. Ko EM, Van Le L (2011) Chemotherapy for gynecologic cancers occurring during pregnancy. Obstet Gynecol Surv 66:291-8

16. Van Calsteren K, Heyns L, De Smet F, Van Eycken L, Gziri MM, Van Gemert W et al (2010) Cancer during pregnancy: an analysis of 215 patients emphasizing the obstetrical and the neonatal outcomes. J Clin Oncol 28: 683-9

17. Koren G, Karey N, Gagnon R, Marxell C, Nulman I, Senikas V (2013) Cancer chemotherapy and pregnancy. J Obstet Gynaecol Can 35:263-80

18. Berry DL, Theriault RL, Holmes FA, Parisi VM, Booser DJ, Singletary SE et al (1999) Management of breast cancer during pregnancy using a standardized protocol. J Clin Oncol 17:855-61

19. Giacalone PL, Laffargue F, Bénos P (1999) Chemotherapy for breast carcinoma during pregnancy: A French national survey. Cancer 86:2266-72

20. Peres RM, Sanseverino MT, Guimarães JL, Coser V, Giuliani L, Moreira RK et al (2001) Assessment of fetal risk associated with exposure to cancer chemotherapy during pregnancy: a multicenter study. Braz J Med Biol Res 34:1551-9

21. Hahn KM, Johnson PH, Gordon N, Kuerer H, Middleton L, Ramirez M et al (2006) Treatment of pregnant breast cancer patients and outcomes of children exposed to chemotherapy in utero. Cancer 107:1219-26

22. Ring AE, Shannon C, Galani E, Ellis PA (2005) Chemotherapy for breast cancer during pregnancy: an 18-year experience from five London teaching hospitals.Chemotherapy for breast cancer during pregnancy: an 18-year experience from five London teaching hospitals. J Clin Oncol 23:4192-7
23. Loibl S, von Minckwitz G, Gwyn K, Ellis P, Blohmer JU, Schlegelberger B et al (2006) Breast carcinoma during pregnancy. International recommendations from an expert meeting. Cancer 106:237-46

24. Amant F, Deckers S, Van Calsteren K, Loibl S, Halaska M, Brepoels L et al (2010) Breast cancer in pregnancy: recommendations of an international consensus meeting. Eur J Cancer 46:3158-68

25. Pregnancy and Breast Cancer. Royal College of Obstetricians and Gynaecologists. https://www.rcog.org.uk/guidelines.

26. Gianni L, Herman EH, Lipshultz SE, Minotti G, Sarvazyan N, Sawyer DB (2008) Anthracycline cardiotoxicity: from bench to bedside. J Clin Oncol 26:3777-84

27. Patanè $S$ (2014) Cardiotoxicity: anthracyclines and long term cancer survivors. Int J Cardiol 176:1326-8

28. Lyu YL, Kerrigan JE, Lin CP, Azarova AM, Tsai YC, Ban Y et al (2007) Topoisomerase 11 beta mediated DNAdouble-strand breaks: implications in doxorubicin cardiotoxicity and prevention by dexrazoxane. Cancer Res 67:8839-46

29. Nikitovic D, Juranek I, Wilks MF, Tzardi M, Tsatsakis A, Tzanakakis GN (2014) Anthracycline-dependent cardiotoxicity and extracellular matrix remodeling. Chest 146:1123-30

30. Siedner $S$, Krüger $M$, Schroeter $M$, Metzler D, Roell W, Fleischmann BK et al (2003) Developmental changes in contractility and sarcomeric proteins from the early embryonic to the adult stage in the mouse heart. J Physiol 548:493-505

31. Van Calsteren K, Verbesselt R, Van Bree R, Heyns L, de Bruijn E, de Hoon J et al (2011) Substantialvariation in transplacental transfer of chemotherapeutic agents in a mouse model. Reprod Sci 18:57-63

32. Van Calsteren $K$, Verbesselt $R$, Beijnen J, Devlieger $R$, De Catte $L$, Chai DC et al (2010) Transplacental transfer of anthracyclines, vinblastine, and hydroxy-cyclophosphamide in a baboon model. Gynecol Oncol 119:594-600

33. Gziri MM, Pokreisz P, De Voz R, Verbeken E, Debiève F, Mertens $L$ et al (2013) Fetal rat hearts do not display acute cardiotoxicity in response to maternal doxorubicin treatment. J Pharmacol Exp Ther 346:362-9

34. Behravan J, Piquette-Miller M (2007) At Drug transport across the placenta, role of the $A B C$ drug efflux transporters. Expert Opin Drug Metab Toxicol 3:819-30

35. Staud F, Cerveny L, Ceckova M (2012) Pharmacotherapy in pregnancy; effect of $A B C$ and SLC transporters on drug transport across the placenta and fetal drug exposure. J Drug Target 20:736-63

36. Syme MR, Paxton JW, Keelan JA (2004) Drug transfer and metabolism by the human placenta. Clin Pharmacokinet 43:487-514

37. Germann N, Goffinet F, Goldwasser F (2004) Anthracyclines during pregnancy: embryo-fetal outcome in 160 patients. Ann Oncol 15:146-50

38. Baumgärtner AK, Oberhoffer $R$, Jacobs VR, Ostermayer $E$, Menzel H, Voigt M et al (2009) Reversible foetal cerebral ventriculomegaly and cardiomyopathy under chemotherapy for maternal AML. Onkologie 32:40-3

39. Achtari C, Hohlfeld P (2000) Cardiotoxic transplacental effect of idarubicin administered during the second trimester of pregnancy. Am J Obster Gynecol 183:511-2

40. Meyer-Wittkopf M, Barth H, Emons G, Schmidt S (2001) Fetal cardiac effects of doxorubicin therapy for carcinoma of the breast during pregnancy: case report and review of the literature. Ultrasound Obstet Gynecol 18:62-6

41. Parodi E, Alluto A, Moggio G, Liberale V, Frigerio M, Sismondi $P$ (2012) Transient ventricular hypocinesia after in utero anthracyclines exposure: a case report and review of the literature. J Matern Fetal Neonatal Med 25:189-92

42. Azim HA, Peccatori FA, Scarfone G, Acaia B, Rossi P, Cascio R et al (2008) Anthracyclines for gestational breast cancer: course and outcome of pregnancy. Ann Oncol 19:1511-2

43. Cardonick E, Gylmandiar D, Somer RA (2012) Maternal and neonatal outcomes of dose-dense chemotherapy for breast cancer in pregnancy. Obstet Gynecol 120:1267-72

44. Gziri MM, Debiève F, De Catte L, Mertens L, Barrea C, Van Calsteren K et al (2012) Chemotherapy during pregnancy: Effects of anthracyclines on fetal and maternal cardiac function. Acta Obstet Gynecol Scand 91:1465-8

45. Zaya MJ, Hines RN, Stevens JC (2006) Epirubicin glucuronidation and UGT2B7 developmental expression. Drug Metab Dispos 34:2097-101

46. Bodner-Adler B, Bodner K, Zeisler H (2007) Breast cancer diagnosed during pregnancy. Anticancer Res 27:1705-7

47. Gadducci A, Cosio S, Fanucchi A, Nardini V, Roncella M, Conte PF et al (2003) Chemotherapy with epirubicin and paclitaxel for breast cancer during pregnancy: case report and review of the literature. Anticancer Res 23:5225-9 
48. Ginopoulos PV, Michail GD, Kourounis GS (2004) Pregnancy associated breast cancer: a case report. Eur J Gynaecol Oncol 25:261-3

49. Peccatori FA, Azim HA Jr, Scarfone G, Gadducci A, Bonazzi C, Gentilini $\mathrm{O}$ et al (2009) Weekly epirubicin in the treatment of gestational breast cancer (GBC). Breast Cancer Res Treat 115:591-4

50. Morrison JL, Botting KJ, Soo PS, McGillick EV, Hiscock J, Zhang S et al (2012) Antenatal steroids and the IUGR fetus: are exposure and physiological effects on the lung and cardiovascular system the same as in normally grown fetuses? J Pregnancy 2012:1-15

51. Kilickap S, Barista I, Akgul E, Aytemir K, Aksoyek S, Aksoy S et al (2005) cTnT can be a useful marker for early detection of anthracycline cardiotoxicity. Ann Oncol 16:798-804

Submit your next manuscript to BioMed Central and we will help you at every step:

- We accept pre-submission inquiries

- Our selector tool helps you to find the most relevant journal

- We provide round the clock customer support

- Convenient online submission

- Thorough peer review

- Inclusion in PubMed and all major indexing services

- Maximum visibility for your research

Submit your manuscript at www.biomedcentral.com/submit
Biomed Central 